\title{
Dual Video Watermarking for CCL Protection and Manipulation Detection
}

\author{
Sung-Won Moon**, Hee-Dong Kim**, Ji-won Lee**, and Heung-Kyu Lee* \\ Division of Web Science Technology* and Dept. of Computer Science** \\ Korea Advanced Institute of Science and Technology (KAIST) \\ 291 Daehak-ro, Yuseong-gu, Daejeon, Republic of Korea \\ Email: \{swmoon, hdkim, jwlee, hklee\}@mmc.kaist.ac.kr
}

\begin{abstract}
Copyright protection issues for personal user's User Generated Contents (UGC) have been arising with the rapid growth of Social Network Services (SNS), blogs, and video sharing services. In this situation, Creative Commons License (CCL) has been established as a convenient method to protect copyrights of UGCs. However, the lack of technical method to protect CCL causes illegal manipulations and sharing of CCL applied UGCs. In this paper, we propose a dual watermarking system which protects copyrights of CCL applied UGCs. Experimental results show that manipulations such as rescaling, cropping, clipping, temporal manipulation, and frame rate changing are detected by semi-fragile watermarks. Simultaneously, robust watermarks notify the CCL information after above manipulations. To prevent interference of two watermarks, robust watermarks are embedded at spatial domain while fragile watermarks are embedded at block based DCT domain.
\end{abstract}

\section{INTRODUCTION}

With the rapid growth of SNS, blog, smart phone, and video sharing services, UGCs are widely spread out on the Internet. As the number of UGCs has been increased, misusing, illegal sharing and manipulation of UGCs has been increased. In this situation, has been established as a convenient method to protect content-creator's copyright CCL [1]. By using CCL, content-creators specify several copyrights on their works. CCL comprises a selection of four basic conditions: Attribution, Non-commercial, No Derivative Works, and Sharealike. Table I shows the meaning and abbreviation of four basic licence conditions. In general, six combinations shown in Table I are used in practice.

TABLE I

FOUR BASIC LICENCE CONDITIONS OF CCL (TOP) WITH SIX AVAILABLE COMBINATIONS (BOTTOM)

\begin{tabular}{c|c|c}
\cline { 2 - 3 } & Abbreviation & Meaning \\
\cline { 2 - 3 } & BY & Attribution \\
& NC & Non-commercial \\
& ND & No Derivative Works \\
& SA & Share-alike \\
\cline { 2 - 3 } & \multicolumn{2}{c}{ Meaning } \\
\hline Abbreviation & Attribution \\
CC BY & \multicolumn{2}{c}{ Attribution Share-alike } \\
CC BY-SA & \multicolumn{2}{c}{ Attribution No Derivative Works } \\
CC BY-ND & \multicolumn{2}{c}{ Attribution Non-commercial } \\
CC BY-NC & \multicolumn{2}{c}{ Attribution Non-commercial Share-alike } \\
CC BY-NC-SA & Attribution Non-commercial No Derivative Works \\
CC BY-NC-ND & \multicolumn{2}{c}{}
\end{tabular}

However, the lack of technical method to protect CCL causes disregard of CCL. To prevent disregard of CCL, removing CCL should be difficult and manipulation of the work should be informed. Digital video watermarking is one of the technical solutions because removing watermarks is hard and embedded watermarks take same manipulation with video. Robust watermarks can be a solution to figure out illegal sharing and existence of CCL because it informs information of the content-creator and CCL even after manipulations [2][5]. Fragile or semi-fragile watermarking is usually used for content authentication since fragile or semi-fragile watermarks cannot be detected when the video has been manipulated [6][9]. In this paper, we exploit dual watermarking system which embeds both robust watermarks and semi-fragile watermarks for CCL protection and manipulation detection.

Dual watermarking system protects copyrights of UGCs in the following scenario. Let's assume a content-creator uploads an UGC with CCL of "BY-ND". This UGC should not be manipulated and users should write the content-creator when they share the UGC. If other user inserts a logo to the UGC and uploads it with CCL of "BY-NC", the system should realize the original CCL, "BY-ND", by detecting robust watermarks. Simultaneously the semi-fragile watermarks inform that whether the video has been manipulated or not. Finally, the system should not allow uploading the manipulated UGC on the Internet.

Kim et al. proposed a hybrid watermarking scheme for CCL applied video contents [10]. They embed both robust and semifragile watermarks in spatial domain to protect CCL applied video's copyrights and to detect manipulation of UGCs. This method is fit to detect global manipulations such as rescaling, clipping, and frame rate changing. Partial manipulation such as logo insertion, however, is hard to be detected with this scheme because semi-fragile watermarks are embedded in spatial domain. Also, since both watermarks are embedded in spatial domain, UGCs have low fidelity after embedding.

In this paper, we propose a dual watermarking scheme which embeds robust watermarks in spatial domain, and semi-fragile watermarks in block based DCT domain. Semifragile watermarks inform manipulations on the video such as rescaling, clipping, frame rate changing, logo insertion, and cropping, while robust watermarks inform the information of content-creators and CCL after those manipulations. 


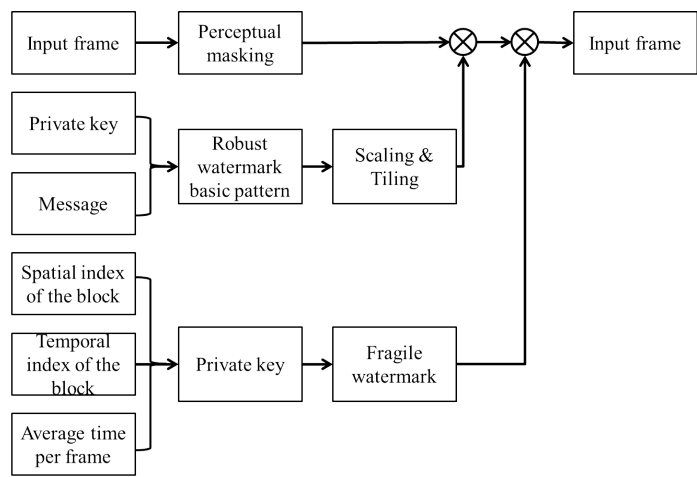

Fig. 1. Embedding process of dual watermarking system

This paper is organized as follows. We explain embedding and detecting processes in Section II and III. Experimental results are given in Section IV and conclusion is presented in Section V.

\section{WATERMARK EMBEDDING}

Figure 1 shows watermark embedding process. The robust watermarks are embedded into spatial domain of video frames and the semi-fragile watermarks are embedded into blockbased DCT domain of video frames.

\section{A. Robust watermark design}

Robust watermarks carrying the information of contentcreators and CCL are designed as follows. Random number generator with private key generates $m \times n$ 2-D basic pattern $W_{b}$. To enhance robustness, the basic pattern $W_{b}$ is enlarged $k$ times where $k$ is calculated by dividing frame height by static length $l$. Then we tiling the enlarged basic pattern $i$ times to horizontally, $j$ times vertically to generate the final pattern $W_{r}$. By embedding different patterns into frames for each time interval $T_{r}$ seconds, we can embed message bits into a target video.

\section{B. Semi-fragile watermark design}

Semi-fragile watermarks detecting manipulation on target videos are designed as follows. In this method, block $B_{i}$ is generated by dividing frames into $N_{w}$ times horizontally and $N_{h}$ times vertically. Each block $B_{i}$ contains semi-fragile watermark $W_{f}$ which follows Gaussian distribution with zero mean and unit variance. Each $W_{f}$ is generated from random number generator using private key made by the following three factors shown in Figure 2. The first factor $K_{1}$ is a spatial index of the block $B_{i}$ and the second factor $K_{2}$ is a temporal index of the frame which contains the block $B_{i}$. The average time per frame is the last factor $K_{3}$ and the private key $K$ is computed with scaling factor to avoid duplication as follows:

$$
K\left(K_{1}, K_{2}, K_{3}\right)=K_{1}+\left(K_{2} \times 10^{3}\right)+K_{3}
$$

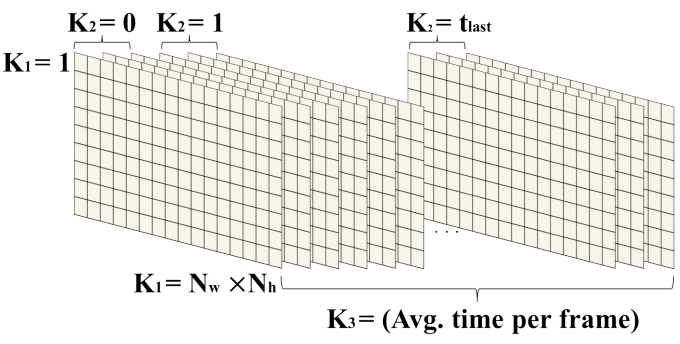

Fig. 2. Private key generation with three factors

\section{Embedding}

Robust watermark pattern $W_{r}$ are embedded into video frames after noise visibility funtion (NVF) masking, motion masking, and luminance masking to maximize imperceptibility and power of the signal. NVF measures the visibility of the noise which is inserted in the frame. Motion masking uses the fact that human visual system (HVS) is insensitive to the noise when the scene has more motions. Luminance masking also uses the characteristic of HVS which is insensitive at brightness changes in darker and brighter region. With consideration of perceptual masking, a robust watermarked frame $X_{r}$ can be expressed as

$$
X_{r}=X+\left(W_{r} \cdot M_{n} \cdot M_{m} \cdot M_{l}\right)
$$

where $X$ is a target frame, $M_{n}$ is the NVF masking filter, $M_{m}$ is a motion masking filter, and $M_{l}$ is a luminance masking filter. Each dot product means pixel-wise product.

After the robust watermarks are embedded, semi-fragile watermarks are embedded in target frames by using blockbased Barni method [11]. First, $b \times b$ DCT is taken for each block $B_{i}$ individually and DCT coefficients are reordered by zigzag scan. Then DCT coefficients from the $(L+1)^{t h}$ to $(L+N)^{t h}$ are taken the sub-watermark $W_{s}$ which is generated by dividing the semi-fragile watermark $W_{f}$. The watermarked coefficient $c_{i}^{w}$ is computed as follows:

$$
c_{i}^{w}=c_{i}+\alpha\left|c_{i}\right| x_{s}
$$

where $c_{i}$ is target coefficient, $\alpha$ is scaling factor of watermark strength, and $x_{s}$ is the value of the embedded watermark $W_{s}$.

\section{WATERMARK DETECTION}

This section provides an overview of watermark detection process to decode a message and determine whether the video has been manipulated or not.

\section{A. Detecting robust watermark}

To extract a message, we use an adaptive Wiener filter as a noise reduction filter because robust watermarks were embedded into a frame as a noise. The estimated watermark $\hat{W}_{r}$ can be extracted by

$$
\hat{W}_{r}(i, j)=X(i, j)-\hat{X}(i, j)
$$


where $X$ is a target frame, $\hat{X}$ is a denoised frame with the adaptive wiener filter, $X(i, j)$ is a pixel value of $X$, and $\hat{X}(i, j)$ is a pixel value of $\hat{X}$. A denoised frame $\hat{X}$ can be calculated as follows:

$$
\hat{X}(i, j)=\mu(i, j)+\frac{\sigma^{2}(i, j)-s^{2}}{\sigma^{2}(i, j)} \cdot((X(i, j)-\mu(i, j))
$$

where $s^{2}$ is the approximated mean of local variance of $X, \mu(i, j)$ is the local mean of $X$, and $\sigma(i, j)$ is the local variance of $X . \hat{W}_{r}$ is calculated by accumulating the estimated watermark patterns for $t_{r}$ seconds and normalizing the accumulated watermark patterns.

To extract messages from robust watermarks, normalized cross correlation(NCC) $Z$ is computed as follows:

$$
Z=\frac{\operatorname{IFFT}\left(F F T\left(\hat{W}_{r}\right) \cdot F F T\left(W_{r}\right)^{*}\right)}{\left|\hat{W}_{r}\right| \cdot\left|W_{r}\right|}
$$

where $W_{r}$ is the reference watermark for the target frame. Embedded robust watermarks are successfully detected when $Z$ is larger than threshold $T_{r}$ defined by:

$$
T_{r}=\mu_{z}+\alpha_{z} \sigma_{z}
$$

where $\mu_{z}$ is the mean of $Z, \sigma_{z}$ is the standard deviation of $Z$, and $\alpha_{z}$ is the controlling factor of false positive error.

\section{B. Detecting semi-fragile watermark}

To determine whether the video has been manipulated or not, we detect $W_{f}$ from each block $B_{i}$ individually. Reference watermark $W_{f}$ is generated by using the private key calculated with the spatial index of $B_{i}, K_{1}$, the temporal index of $B_{i}$, $K_{2}$, and the average time per frame $K_{3}$. The watermarked coefficients $c_{i}^{w}$ are calculated by applying $b \times b$ DCT to each block $B_{i}$. DCT coefficients $c_{i}^{w}$ from the $(L+1)^{t h}$ to $(L+N)^{t h}$ are taken to construct a vector $C=\left\{c_{1}^{w}, c_{2}^{w}, \ldots, c_{N}^{w}\right\}$. We compute the correlation between the vector $C$ and the reference watermark $W_{f}$. The correlation is computed as follows:

$$
\operatorname{Corr}\left(W_{f}, C\right)=\frac{W_{f} \cdot C}{N}
$$

To decide the watermarked video is manipulated or not, the correlation is compared to a threshold $T_{f}$. The threshold $T_{f}$ is given by:

$$
T_{f}=\frac{\alpha_{f}}{3 N} \sum_{i=1}^{N}\left|c_{i}^{w}\right|
$$

We decide that a target frame has been manipulated when more than $P_{f}$ percentages of blocks in the target frame have been manipulated.

We decide whether a target video has been manipulated by following steps:

- Step 1 : Detect semi-fragile watermark $W_{f}$ from frame group. Go to step 2.
TABLE II

PSNR AFTER EMBEDDING WATERMARKS

\begin{tabular}{c|c|c|c||c}
\hline & $640 \times 360$ & $1280 \times 720$ & $1920 \times 1080$ & Kim [10] \\
\hline PSNR(dB) & 44.7 & 44.3 & 44.5 & 43.4 \\
\hline
\end{tabular}

- Step 2 : If more than $P_{\text {global }}$ percentages of frames in frame group are manipulated, we decide that target video has been manipulated. If not, go to step 3 .

- Step 3 : If more than $P_{\text {partial }}$ percentages of blocks in frame group with same spatial index are manipulated, we decide that target video has been manipulated. If not, go to step 4.

- Step 4 : We decide the target video has not been manipulated.

\section{EXPERIMENTAL RESUltS}

In order to evaluate the proposed watermarking system, 15 video clips of 5-minute length were used for test. Resolution of each 5 video clips were $640 \times 360,1280 \times 720$, and $1920 \times 1080$, and frame rate was $23.93 \mathrm{fps}$. A message of $40 \mathrm{bit}-$ length including 3 bits for CCL information was embedded. The target video was encoded with bit rate $1000 \mathrm{kbps}$ for $640 \times 360$ video resolution, $2000 \mathrm{kbps}$ for $1280 \times 720$ video resolution, and 3000kbps for $1920 \times 1080$ video resolution after embedding watermarks. Chosen resolution and bit rate are widely used on the Internet such as YouTube.

\section{A. Fidelity test}

Table II shows that average peak signal to noise ratio (PSNR) for test videos are higher than 44dB. It means that embedded watermarks satisfy imperceptibility for HVS. Table II also show that proposed watermarks have better fidelity on watermarked video than Kim's scheme [10].

\section{B. Robustness test}

Table III shows that embedded message bits including CCL information are successfully extracted after following manipulation:

- Scaling : scaling the test videos to its half resolution.

- Cropping : cropping the test videos with 16:9 picture ratio to $4: 3$ picture ratio.

- Clipping : clipping the first 20seconds of the test videos.

- Logo insertion : inserting a logo which is smaller than $5 \%$ of frame size to the test videos.

TABLE III

RESULTS OF EXTRACTING MESSAGE FROM TEST VIDEO (O MEANS THAT ALL 40BITS OF MESSAGE ARE EXTRACTED SUCCESSFULLY)

\begin{tabular}{c||c|c|c}
\hline Manipulation type & $640 \times 360$ & $1280 \times 720$ & $1920 \times 1080$ \\
\hline No manipulation & $\mathrm{O}$ & $\mathrm{O}$ & $\mathrm{O}$ \\
Rescaling & $\mathrm{O}$ & $\mathrm{O}$ & $\mathrm{O}$ \\
Cropping & $\mathrm{O}$ & $\mathrm{O}$ & $\mathrm{O}$ \\
Clipping & $\mathrm{O}$ & $\mathrm{O}$ & $\mathrm{O}$ \\
Logo insertion & $\mathrm{O}$ & $\mathrm{O}$ & $\mathrm{O}$ \\
Frame rate change & $\mathrm{O}$ & $\mathrm{O}$ & $\mathrm{O}$ \\
\hline
\end{tabular}


TABLE IV

RESULTS OF FRAGILITY TEST FOR NON-MANIPULATED VIDEOS (TOP) AND MANIPULATED VIDEOS (BOTTOM)

\begin{tabular}{c||c|c|c}
\hline & $640 \times 360$ & $1280 \times 720$ & $1920 \times 1080$ \\
\hline \# of manipulated video & 0 & 0 & 0 \\
\hline \multicolumn{3}{|c}{} \\
\hline Manipulation type & Avg. manipulation rate $(\%)$ & Manipulation \\
\hline Rescaling & 96.50 & $\mathrm{O}$ \\
Cropping & 96.56 & $\mathrm{O}$ \\
Clipping & 96.11 & $\mathrm{O}$ \\
Frame rate change & 98.95 & $\mathrm{O}$ \\
Logo insertion & - & $\mathrm{O}$ \\
\hline
\end{tabular}

- Frame rate change : changing the frame rate of the test videos from $23.98 \mathrm{fps}$ to $24 \mathrm{fps}$.

\section{Fragility test}

For fragility test, the parameters were set to $b=8, L=10$, $N=1, \alpha=\alpha_{f}=0.2, N_{w}=16, P_{f}=50, P_{\text {global }}=90$, $P_{\text {patial }}=100, N_{h}=9$, and the frame group is constructed with 450 frames. Same manipulations in robustness test were taken for the test videos. Table IV shows that no frame group has been decided as manipulated frame group. Table IV also shows that manipulation detection rate is over $96.0 \%$ for target frames. Because logo insertion manipulates same region of frame continuously, manipulation can be detected by step 3 of fragile watermark detection. All manipulated videos by manipulations mentioned above are decided as a manipulated video by fragility test.

\section{CONCLUSION}

In this paper, we have proposed a dual watermarking system for CCL protection and manipulation detection. To protect content-creator's copyrights, CCL information and integrity of target video should be protected. Experimental results shows that we can detect various manipulations while we can extract 40-bit message with CCL information from target videos with various resolution. Also, proposed watermarks are imperceptible for HVS and guarantees better fidelity than previous scheme.

In future research, we will try to use discrete wavelet transform(DWT) instead of DCT to improve robustness of semi-fragile watermark under video compression. Also, we can change the length of sub-watermark to find it's optimal length.

\section{ACKNOWLEDGMENT}

This research was supported by WCU (World Class University) program and NRL(National Research Program) under the National Research Foundation of Korea and funded by the Ministry of Eduation, Science and Technology of Korea (Project No: R31-30007) and (No. R0A-2007-000-20023-0)

\section{REFERENCES}

[1] Creative Commons (Online), Available: http://creativecommons.org/

[2] I. J. Cox, J. Kilian, F. T. Leighton, and T. Shamoon, "Secure spread spectrum watermarking for multimedia,"IEEE Trans. on Image Processing, vol. 6, no. 12, pp. 1673-1687, 1997.
[3] M. Barni, F. Bartolini, V. Cappellini, and A. Piva, "A DCT-domain system for robust image watermarking," Signal Processing, vol. 66, pp. 357-372, 1998.

[4] K-S. Kim, H-Y Lee, D-H. Im, and H-K. Lee, "Practical, real-time and robust watermarking on the spatial domain for high-definition video contents," IEICE Trans. on Inf. Syst., vol. E91-D, pp.1359-1368, 2008.

[5] G. Qiu, P. Maziliano, A. T. S. Ho, D. HE, and Q. Sun, "A hybrid watermarking scheme for h.264/avc video," in Proc. ICPR(International Conference on Pattern Recognition, vol. 4, pp.865-869, 2004

[6] J. Fridrich, "Security of fragile authentication watermarks with localization," in Proc. SPIE, vol. 4675, pp.691-700, 2002.

[7] C-Y. Lin and S-F. Chang, "Semifragile watermarking for authenticating jpeg visual content," in Proc.SPIE, vol. 5681, pp.353-362, 2005.

[8] S. Thiemert, H. Sahbi, and M. Steinebach, "Applying interest operators in semi-fragile video authentication," in Proc. SPIE, vol. 5681, pp.353362,2005

[9] C-C. Wang and Y-C. Hsu, "Fragile watermarking for h.264 video stream authentication," Intelligent Systems Design and Applications (ISDA), vol. 1, pp.77-80, 2008.

[10] H-D. Kim, T-W. Oh, J-W. Lee, and H-K. Lee, "A hybrid watermarking scheme for CCL-applied video contents," in Proc. 3rd European Workshop on Visual Information Processing, Paris, France, pp. 199-204, 2011.

[11] K. Al-Dhmani, M.E. Al-Mualla, "A block-based Barni watermarking method for improved robustness under video compression attacks," in Proc. GCC Conference (GCC), pp.1-5, 2006. 